\title{
How to Assess Patients with Rheumatoid Arthritis and Concomitant Fibromyalgia?
}
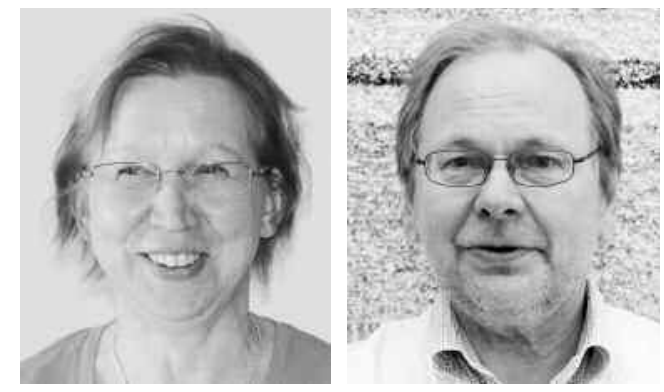

Rheumatoid arthritis (RA) is a progressive autoimmune disorder, and fibromyalgia (FM) is a pain syndrome due to dysregulation of central pain processing. Both syndromes predominantly affect females. Further, although a patient with either disorder may be any age, most patients are 50 years and above. There is abundant clinical experience that patients with rheumatic disorders including systemic lupus erythematosus, Sjögren's syndrome, and RA have simultaneous FM more often than a control population ${ }^{1}$.

Chronic widespread pain, the hallmark of FM, is prevalent among elderly women in the general population and appears to predict progression of disability ${ }^{2}$, and persons fulfilling FM criteria show impaired functioning and quality of life $^{3}$. Moreover, Wolfe and Michaud ${ }^{4}$ have reported that patients with RA and FM (RAF) have more severe RA by both subjective and objective measures, worse outcomes, and lower quality of life compared to other RA patients.

Patients with RAF and patients with other autoimmune inflammatory disease and concurrent FM may be misdiagnosed and, more important, their therapies may be mistargeted. Keeping in mind that both rheumatoid factor (RF) and antinuclear antibody tests are prevalently false-positive in elderly patients ${ }^{5}$, the appropriate diagnosis is of vital importance regarding the choice of effective and safe therapies.

Assessment of inflammatory disease activity using disease activity indices has emerged as the most promising way to judge the success of therapies in daily clinical care as well as in formally conducted (drug) trials. The Disease Activity Score (DAS) and its simplified version DAS28, which is based on 28-joint count including number of swollen (SJC) and tender joints (TJC), patient general well-being by 100$\mathrm{mm}$ visual analog scale, and acute-phase reactant (either erythrocyte sedimentation rate, ESR, or C-reactive protein) ${ }^{6}$, have become the generally accepted standard to measure clinical disease activity. Indeed, authorities and payers in many countries have accepted the use of this tool for allocating new, expensive biological therapies to patients.

As outlined above, assessment of RA disease activity is a challenge, because features such as fatigue, pain, and tender joints are common not only in RA, but also in other diseases, and even in elderly subjects in the general population ${ }^{7}$. Thus, use of the DAS28 as a disease activity measure includes well known pitfalls ${ }^{8}$. In particular, one has to be careful when applying these indices in patients with chronic pain syndromes like FM.

Pain and patient gender form the main determinants for the DAS28 score, with female patients with RA having higher DAS28 values than male cases ${ }^{9}$. Consequently, DAS28 remission (DAS28 < 2.6) is more often achieved by male than female RA patients ${ }^{10}$. The same gender difference also concerns newer disease activity indices like the Simplified Disease Activity Index (SDAI) ${ }^{11}$ and Clinical Disease Activity Index $(\mathrm{CDAI})^{12}$. The values of SDAI and CDAI are also highly dependent on the patient's pain perception ${ }^{13}$. However, the cutoff points for low, moderate, and high disease activity and remission are the same for all, independent of the patient's age, gender, or optional comorbidities.

The American College of Rheumatology remission criteria are stricter than others listed above because they include requirements of no pain and no tender joints ${ }^{14}$. Thus, it appears plausible, irrespective of the disease activity tool in use, that remission is less often achieved in RAF patients compared to other RA patients.

Nevertheless, the rheumatologic community has decided that the goal of treatment in RA should be remission in all patients irrespective of age, gender, or comorbidies including pain syndromes (like FM). By now it is well known that remission is best achieved by tight disease control and by proper use of disease activity indices to guide therapies ${ }^{15-17}$. Indeed, composite scores of disease activity such as DAS28 are of great value in evaluation of treatment response in RA clinical trials. However, composite indices that work well in a group may not do so on an individual level ${ }^{8}$.

Leeb, et $\mathrm{l}^{18}$ compared use of DAS28 in $62 \mathrm{RA}$ and 26 FM patients, with no difference in total DAS28 scores found

See RA and FM: a frequent unrelated association complicating disease management, page 58 Personal non-commercial use only. The Journal of Rheumatology Copyright @ 2009 . All rights reserved. 
between these groups. Since FM is a noninflammatory disorder, the ESR and SJC were within normal range, and high DAS28 scores were based on poorly perceived global health and high numbers of TJC. The authors concluded that DAS28 values for expressing disease activity in patients with RA may be flawed due to coexisting FM and should therefore be regarded with caution, as high pain levels lead to higher total scores.

In this issue of The Journal Coury, et al report a study ${ }^{19}$ comparing several clinical and sociodemographic variables between 105 patients with RA, 49 with RAF, and 28 with FM; all were female. Their special interest was to find out whether FM might be a marker of severe RA. The results were ambiguous.

Although the patients with RA and RAF were comparable for age, disease duration, RF, antifilaggrin antibody positivity, comorbid diseases, and surgical history, the number of general practitioner consultations for reasons other than RA was significantly greater in the RAF population. More important, in accord with results of Wolfe and Michaud ${ }^{4}$, perceived physical function (by Health Assessment Questionnaire) and quality of life (by Medical Outcome Study Short Form-36) were significantly poorer in RAF versus RA patients. The results also demonstrate that clinical disease activity (by DAS28) is higher in RAF than in RA patients. In accord with the study of Leeb and coworkers ${ }^{18}$ DAS28 levels were comparable between RA and FM populations. Unexpectedly, however, the radiographic extent of tissue damage in peripheral joints was statistically significantly higher in the $76 \mathrm{RA}$ than in the $36 \mathrm{RAF}$ patients, whose radiographs were available. It is reasonable to ask why?

RAF patients had significantly longer duration of morning stiffness as well as higher TJC and SJC than the RA cases. Higher SJC in RAF patients, however, were due to swelling in metatarsophalangeal joints, which are difficult to assess, especially in obese patients (RAF patients had higher body mass index vs RA patients). Knowing that RAF patients had less erosive changes in their peripheral joints, observed swelling in these cases most probably was not due to inflammation. Indeed, preliminary information suggests that obesity may protect RA patients from joint destruction $^{20}$. On the other hand, higher TJC in patients with RAF can easily be explained by FM. Since joints of ankles and feet are not included in the DAS28, the higher DAS28 values of RAF patients in this study can be accounted for by higher TJC and poorer perceived general health. It is known that pain, the hallmark of FM, has a strong correlation with general health ${ }^{21}$.

What can be done to avoid the overestimation of disease activity in patients with RAF? Unfortunately, cursory clinical examination with an extensive battery of diagnostic testing has replaced proper clinical examinations. Clinicians who are comfortable with the diagnosis of FM, who perform thorough history-taking and clinical examination including the pain threshold estimation (tender point examination), and interpret laboratory tests with caution, are more likely to avoid diagnostic errors ${ }^{5}$. Plain radiographs of hands and feet should be monitored, and in the RAF patient group newer imaging tools like power Doppler ultrasound and magnetic resonance imaging, limited by their availability, may also be helpful to show whether pain is due to synovial inflammation. Nevertheless, concomitant FM should be remembered when treatment response in RA seems to be inadequate. This applies especially to elderly female patients with RA. It is expensive, and pointless, to treat FM pain with biologic therapies.

It is easy to agree with Coury, et $a l^{19}$ that the association of RA and FM is not a marker of worse prognosis with respect to tissue destruction. On the other hand, function and quality of life are characteristics valued by all. In that respect, FM worsens the prognosis of the RAF population. Further, in contrast to Coury, et al's assumption, the association of the 2 conditions may not be random, since RA patients may be prone to develop FM due to chronic painful input into the central nervous system. Finally, composite indices are good tools when assessing and designing treatment of RA patients, but laboratory tests, imaging, or composite indices can never substitute for careful assessment of disease activity by an experienced clinician.

\footnotetext{
HEIDI MÄKINEN, MD, Tampere University Hospital, Tampere;

PEKKA HANNONEN, MD, $\mathrm{PhD}$, Department of Rheumatology, Jyväskylä Central Hospital, Jyväskylä, Finland.
}

Address reprint requests to Dr. H. Mäkinen, Tampere University, PL 2000, 33521 Tampere, Finland.

\section{REFERENCES}

1. Gran JT. The epidemiology of chronic generalized musculoskeletal pain. Best Pract Res Clin Rheumatol 2003;17:547-61.

2. Leveille SG, Ling S, Hochberg MC, et al. Widespread musculoskeletal pain and the progression of disability in older disabled women. Ann Intern Med 2001;135:1038-46.

3. Mas AJ, Carmona L, Valverde M, Ribas B. Prevalence and impact of fibromyalgia on function and quality of life in individuals from the general population: results from a nationwide study in Spain. Clin Exp Rheumatol 2008;26:519-26.

4. Wolfe F, Michaud K. Severe rheumatoid arthritis (RA), worse outcomes, comorbid illness, and sociodemographic disadvantage characterize RA patients with fibromyalgia. J Rheumatol 2004;31:695-700.

5. Blumenthal DE. Tired, aching, ANA-positive: does your patient have lupus or fibromyalgia? Cleve Clin J Med 2002;69:143-6; 151-2.

6. van der Heijde DM, van 't Hof MA, van Riel PL, et al. Judging disease activity in clinical practice in rheumatoid arthritis: first step in the development of a disease activity score. Ann Rheum Dis 1990;49:916-20. 
7. Sokka T, Mäkinen H, Hannonen P, Pincus T. Most people over age 50 in the general population do not meet ACR remission criteria or OMERACT minimal disease activity criteria for rheumatoid arthritis. Rheumatology Oxford 2007;46:1020-3.

8. Makinen H, Kautiainen H, Hannonen P, et al. Disease Activity Score 28 as an instrument to measure disease activity in patients with early rheumatoid arthritis. J Rheumatol 2007;34:1987-91.

9. Leeb BF, Haindl PM, Maktari A, Nothnagl T, Rintelen B. Disease Activity Score-28 values differ considerably depending on patient's pain perception and sex. J Rheumatol 2007;34:2382-7.

10. Forslind K, Hafstrom I, Ahlmen M, Svensson B. Sex: a major predictor of remission in early rheumatoid arthritis? Ann Rheum Dis 2007;66:46-52.

11. Smolen JS, Breedveld FC, Schiff MH, et al. A simplified disease activity index for rheumatoid arthritis for use in clinical practice. Rheumatology Oxford 2003;42:244-57.

12. Aletaha D, Nell VP, Stamm T, et al. Acute phase reactants add little to composite disease activity indices for rheumatoid arthritis: validation of a clinical activity score. Arthritis Res Ther 2005;7:R796-806.

13. Rintelen B, Haindl PM, Maktari A, Nothnagl T, Hartl E, Leeb BF. SDAI/CDAI levels in rheumatoid arthritis patients are highly dependent on patient's pain perception and gender. Scand J Rheumatol 2008;29:1-4.

14. Pinals RS, Masi AT, Larsen RA. Preliminary criteria for clinical remission in rheumatoid arthritis. Arthritis Rheum 1981;24:1308-15.
15. Grigor C, Capell H, Stirling A, et al. Effect of a treatment strategy of tight control for rheumatoid arthritis (the TICORA study): a single-blind randomised controlled trial. Lancet 2004;364:263-9.

16. Goekoop-Ruiterman YP, de Vries-Bouwstra JK, Allaart CF, et al. Clinical and radiographic outcomes of four different treatment strategies in patients with early rheumatoid arthritis (the BeSt study): a randomized, controlled trial. Arthritis Rheum 2005;52:3381-90.

17. Fransen J, Moens HB, Speyer I, van Riel PL. Effectiveness of systematic monitoring of rheumatoid arthritis disease activity in daily practice: a multicentre, cluster randomised controlled trial. Ann Rheum Dis 2005;64:1294-8.

18. Leeb BF, Andel I, Sautner J, Nothnagl T, Rintelen B. The DAS28 in rheumatoid arthritis and fibromyalgia patients. Rheumatology Oxford 2004;43:1504-7.

19. Coury F, Rossat A, Tebib A, et al. Rheumatoid arthritis and fibromyalgia: a frequent unrelated association complicating disease management. J Rheumatol 2009;36:58-62.

20. van der Helm-van Mil AH, Breedveld FC, Huizinga TW. Aspects of early arthritis. Definition of disease states in early arthritis: remission versus minimal disease activity [review]. Arthritis Res Ther 2006;8:216.

21. Smedstad LM, Kvien TK, Moum T, Vaglum P. Correlates of patients' global assessment of arthritis impact. A 2-year study of 216 patients with RA. Scand J Rheumatol 1997;26:259-65.

J Rheumatol 2009;36:9-11; doi:10.3899/jrheum.081100 